\title{
Has physics come to an end?
}

\author{
The idea that physics has almost been worked out as a field for interesting investigations is widely current but \\ mistaken. There is as much left to do as has been done.
}

New Delhi. What is there left in physics? That was the plaintive question this week from a score of New Delhi University's physics faculty, worrying about the difficulty of teaching students anything of value without modern or even adequate equipment. One of their concerns is that even the brightest students, starved of stimulation, lose heart and drift off to other occupations. Another is that they themselves, confined to theoretical studies by shortages of equipment and deprived of the stimulation of stimulated students, are understandably unsure about their subject. The news of the abandonment of the Superconducting Super Collider, which has travelled fast, has not much helped. It is a fair guess that the same lack of spirit is to be found in better provided places.

But, with all the extenuating circumstances, the question resonates uncomfortably with triumphalist statements from the likes of Stephen Hawking that the "theory of everything" is within reach. What is there left in physics, in all ways the field of enquiry from which reductionists would derive the properties of the remainder of the natural world, but which sometimes has the appearance of being a cornfield from which most of the crop has been harvested? The roster of the great discoveries of the past century accentuates present ennui: X-rays and radioactivity, electrons, cosmic rays, special relativity, quantum theory, general relativity, electron spin, mesons, fission, thermonuclear fusion, nucleogenesis, neutron stars, palaeomagnetism and plate tectonics, masers, lasers and transistors are each sufficient to make the shadowy top quark and Higgs boson seem minor events in such a calendar. A decade ago, there was a chance that the discovery of high-temperature superconductivity might have brought physics to life; it may yet, when somebody is able to account for it convincingly.

Matters inevitably seem worse because the comparison with parts of biology is unavoidable. What physicist would dare quarrel with the view that molecular genetics is where the action is and that, quite apart from the practical value of a listing of the human genome, the comparison between that and other genomes will surely provide such a detailed understanding of the intricacies of evolution that it will be possible to reconstruct the history of life on the Earth, even back to the beginning. Physicists cannot fail to have noticed the attention the newspapers pay to this prospect. Those who remember the cheerful decades before 1970 will be doubly downcast.

In one sense, of course, physicists have brought these troubles on themselves. They have been ascetic to a fault. Faced with, say, the emergence of semiconductor devices, they have taught the essence of solid-state physics and then sent interested students off to departments of electrical or electronic engineering to make use of what they have learned. (It is a lucky accident that the scanning tunnelling microscope has made the structure of the surfaces of silicon and other metals, with or without absorbents, a part of physics.) Palaeomagnetism has similarly been hived off to geophysics, radioastronomy to separate installations sometimes called observatories, much of fluid dynamics to geophysics (and the remainder to meteorology) and interesting questions such as nucleogenesis and interstellar chemistry to departments of astrophysics.

The reasons for this spawning of new disciplines from physics are plain and practical. They have to do with teaching. The hard core of the curriculum is demanding enough, from mechanics (classical and otherwise) and thermodynamics to optics and electromagnetism. And students must acquire that distinctive blend of knowing how to formulate problems accurately and of how to approximate the solutions of problems they cannot solve. The skill of designing an experiment that will actually measure what is intended is the essence of the craft. Stripped of the fun and games, it is bound to seem a little spartan, even dull. Maybe that is how Delhi students find it, but they are unlikely to be alone.

But none of this implies that physics is dead; it merely seems like that to some. The most casual glance through the specialist journals will show, for example, that the ingenuity now in evidence is more remarkable than it has ever been. The flurry of excitement, a few years ago, about the supposed 'fifth force' is a splendid proof that the traditions of elegant design are alive and well (see Fischbach, E. \& Talmadge, C. Nature 356, 207-215; 1992). The ingenuity lavished on the undeserving claims of cold fusion (see, for example, Williams, D. E. et al. Nature 342, 375-384; 1989) is another proof of that. But, nowadays, the elegant experiments may consume years in preparation and execution. And then they are rarely as significant as, say, the Michelson-Morley experiment (for all the controversy its interpretation provoked).
Indeed, there now seems only a tenuous connection between human-scale laboratory experiments and the grand themes of physics - the structure of the Universe and the nature of the objects, made of particles, that it contains. (The hunts for bare quarks, sometimes with suitably instrumented domestic vacuum cleaners, and for magnetic monopoles, have been at least temporarily abandoned.) The small armies working around particle detectors are no longer on a human scale.

Even so, the experiments in the journals are not unconnected with the old grand themes. There is still, for example, much to be done to pin down quantum nonlocality (or to make actual the Einstein-PodolskyRosen gedanken experiment of 1935) and to verify that cyclic variations of some parameter characterizing a quantum system may change its quantum phase and thus its state. Even the meaning of the concept of a quantum state is now up for grabs experimentally, thanks to the ingenuity with which single atoms (or a few) can be physically trapped and then manipulated with lasers. It is no wonder that laser atomic physics is now a happy hunting-ground for experimentalists. It cannot be long before the transport of electrons through carriers of narrow dimensions (called 'quantum wires') follows suit.

But then, of course, there is a whole host of other gaps in understanding that cry out for attention. What about the quantization of the gravitational field, for example? In what sense can physics be said to be complete while such issues are wide open? If it comes to that, what is to be made of the puzzle over dark matter and the possibly connected difficulty about the Sun's underproduction of neutrinos? As always in physics, such gaps in understanding point to what are probably huge fields of intellectual activity still unexplored.

That is the sense in which it is especially misleading to think of the missing quark and the Higgs boson as the capstones that will bring physics to an end. The most likely outcome of the accelerator experiments being planned is either that these particles will not be found at all or that something else will appear instead. And then, of course, in the most constructive way, the field will come to life again. For a long time, the idea that there is a theory of everything will be forgotten. There will be no brownie points for wondering what it may be. Physics, in other words, is far from dead. John Maddox 